\title{
Dispersed gold nanoparticles potentially ruin gold barley yellow dwarf virus and eliminate virus infectivity hazards
}

\author{
Noorah A. Alkubaisi ${ }^{1} \cdot$ Nagwa M. A. Aref ${ }^{1}$
}

Received: 4 August 2016/Accepted: 12 October 2016/Published online: 25 October 2016

(C) The Author(s) 2016. This article is published with open access at Springerlink.com

\begin{abstract}
Gold nanoparticles (AuNPs) application melted barley yellow dwarf virus-PAV (BYDV-PAV) spherical nanoparticle capsids. Synergistic therapeutic effects for plant virus resistance were induced by interaction with binding units of prepared AuNPs in a water solution which was characterized and evaluated by zeta sizer, zeta potential and transmission electron microscopy (TEM). The yield of purified nanoparticles of BYDV-PAV was obtained from Hordeum vulgare (Barley) cultivars, local and Giza 121/Justo. It was $0.62 \mathrm{mg} / \mathrm{ml}$ from $27.30 \mathrm{~g}$ of infected leaves at an A260/A280 ratio. Virus nanoparticle has a spherical shape $30 \mathrm{~nm}$ in size by TEM. BYDV-PAV combined with AuNPs to challenge virus function in vivo and in vitro. Dual AuNPs existence in vivo and in vitro affected compacted configuration of viral capsid protein in the interior surface of capsomers, the outer surface, or between the interface of coat protein subunits for 24 and $48 \mathrm{~h}$ incubation period in vitro at room temperature. The sizes of AuNPs that had a potentially dramatic deteriorated effect are 3.151 and $31.67 \mathrm{~nm}$ with a different intensity of $75.3 \%$ for the former and $24.7 \%$ for the latter, which enhances optical sensing applications to eliminate virus infectivity. Damages of capsid protein due to AuNPs on the surface of virus subunits caused variable performance in four different types of TEM named puffed, deteriorated and
\end{abstract}

Nagwa M. A. Aref

Narif@ksu.edu.sa; dr.nagwaref@gmail.com

Noorah A. Alkubaisi

Nalkubaisi@ksu.edu.sa

1 Department of Botany and Microbiology, College of Science, Female Scientific and Medical Colleges, King Saud University, P.O. Box 22452, Riyadh 11495, Kingdom of Saudi Arabia decorated, ruined and vanished. Viral yield showed remarkably high-intensity degree of particle symmetry and uniformity in the local cultivar greater than in Giza $121 /$ Justo cultivar. A high yield of ruined VLPs in the local cultivar than Justo cultivar was noticed. AuNPs indicated complete lysed VLPs and some deteriorated VLPs at $48 \mathrm{~h}$.

Keywords Dispersed gold nanoparticles · Gold barley yellow dwarf virus - Plasmonic resonant energy · Agrinanotechnology $\cdot$ Viral biotemplates · Optical sensing · Optical scattering energy $\cdot$ AuNPs optical antennas

\section{Introduction}

Nanotechnology is a multilateral term for a broad range of relatively novel technologies; the main unifying theme is that it is concerned with the matter on the nanometer scale (Greek ñanos means dwarf). It is a highly multidisciplinary area that describes a field of applied science and technology focused on the synthesis, characterization and application of materials on this scale (Steinmetz and Evans 2007). It has become a prominent industrial and scientific field, with increasing production and wider applications that are expected to become routinely present in natural ecosystems. Application of nanomaterial-based agrochemicals could lead to widespread intentional environmental dispersion (Hassellöv et al. 2008). The successful application of nano-platforms in medicine under in vitro conditions has generated some interest in agri-nanotechnology which held the promise of agrochemicals and sitetargeted delivery of different macromolecules needed for plant disease resistance (Nair et al. 2010). Growing nanoscale disease-resistant varieties as natural substitution agents in our agriculture environments is the most 
economical and efficient method of controlling diseases. Resistance to BYDV is of great significance. To protect the plant against the virus, the FAO/WHO meeting in 2011 summarized major issues of nanomaterial applications in crop protection as follows: accurate characterization of nanomaterials in biological matrices for in-depth understanding, nanomaterial interaction with biology, dose-response considerations and exposure assessment and characterization studies. Giljohann et al. 2010 proved the advantage of some properties of modified AuNPs with different surface chemistries such as nucleic acids and peptides, and each kind of chemistry endows the particles with different properties. Virus-based nanotechnology has generated interest in some usage due to the specificity of virus interaction design with inorganic and organic nanoparticles, in addition to multifunctional proteinaceous shell (capsid) structure surrounding the genomic material (Capek 2015). Plant viruses are a valuable addition to the bionanotechnology toolbox for the construction of new materials for use in medical applications. There is currently a knowledgeable interest in the self-assembly of composite materials from metal nanoparticle, proteins and nucleic acids (Niemeyer 2001). DNA molecules, in particular, play a major role in this approach due to their large molecular recognition capabilities (Niemeyer et al. 2003). Nanoconjugates with noble metal nanoparticles have ideal template construction due to the nanosized components of the virus particles, monodispersity, and a variety of chemical groups available for modification. Multifunctional viruses integrated with nanoparticles and other functional additives to generate bioconjugates with different properties-possible antiviral and antibacterial activities-viral nanoparticles, noble metals, and decorated viruses and their nanoconjugates. Successful development of ultrasensitive constructs for bioimaging is a challenging function such as wellcharacterized fine three-dimensional structures, multivalency, orthogonal reactivities, and responsiveness to genetic modifications. Imaging and binding units can find out the alteration tolerances on the surface of protein subunits, which leads to deteriorated VLPs by gold nanoparticles. These findings highlight some recent progress in the applications of viruses in bioimaging in TEM (Li et al. 2010).

\section{Materials and methods}

\section{Preparing AuNPs in water solution and TEM examination}

AuNPs were prepared by using citrate ion as a reducing agent. This process was conducted to produce modestly monodispersed spherical AuNPs which were suspended in water, according to Alkubaisi et al. (2015) and Turkevich et al. (1951). A drop of this particle placed on a carboncoated Formvar grid for 2 min and then examined by TEM with a JOEL 1220.

\section{Characterization of AuNPs according to zeta sizer and zeta potential and BYDV-PAV particles}

AuNPs were tested for size distribution that was reported by intensity using zeta sizer for Malvern (ZEN3600). Then, the magnitude of the electrostatic or charge repulsion/attraction between the particles was measured by zeta potential which reflects the properties of their effective charge. AuNPs were examined in TEM which obtained from purified crude sap samples of treated barley with AuNPs only.

\section{AuNPs treatment with characterized BYDV-PAV}

The purified virus particles were obtained from the infected barley according to Alkubaisi et al. (2015). The partially purified sap was stained using uranyl acetate and examined in TEM. Then, the barley was double treated with both characterized AuNPs and BYDV-PAV in two kinds of Hordeum vulgare cultivars, local and Giza 121/Justo. Both cultivars were obtained from the College of Agriculture, Plant Protection Department, King Saud University. AuNPs were applied first as a protection pre-treatment in vivo on barley plant, followed by purified BYDV-PAV particles (AuNPs/BYDV-PAV), while purified BYDVPAV particles were applied first on both cultivars followed by AuNPs as a post-treatment in vivo (BYDV-PAV/ AuNPs) for comparative efficacy for AuNPs.

\section{Dual AuNPs effects for 24 and $48 \mathrm{~h}$ in vitro by TEM examination}

The purified virus particles were obtained from previously treated barley according to Alkubaisi et al. (2015). Then, these were instantly incubated with AuNPs for 24 and $48 \mathrm{~h}$ in vitro at room temperature, and then five grids from each similar treatment of AuNPs for both cultivars and time incubation as illustrated in the following abbreviations for each one were negatively stained with uranyl acetate and examined in TEM. Partially Purified sap from the local cultivar was treated with AuNPs/infected by BYDV-PAV and then treated again in vitro by AuNPs for 24 and $48 \mathrm{~h}$ and coded as:

1. Local-AuNPs/BYDV-PAV (NPs in vivo)-AuNPs24 vs $48 \mathrm{~h}$ (NPs in vitro).

Partially purified sap from the Justo cultivar that was obtained by treatment with AuNPs/infected by BYDV- 
PAV and then treated again in vitro by AuNPs for 24 and 48 h was coded as:

2. Justo-AuNPs/BYDV-PAV (NPs in vivo)-AuNPs24 vs 48 h (NPs in vitro).

Partially Purified sap from the local cultivar was treated with AuNPs/infected by BYDV-PAV and then treated again in vitro by AuNPs for 24 and $48 \mathrm{~h}$ and coded as:

3. Local-BYDV-PAV/AuNPs (NPs in vivo)-AuNPs$24 \mathrm{~h}$ (NPs in vitro).

Partially Purified sap from the local cultivar was treated with AuNPs/infected by BYDV-PAV and then treated again in vitro by AuNPs for 24 and $48 \mathrm{~h}$ and coded as:

4. Local-BYDV-PAV/AuNPs (NPs in vivo)-AuNPs$48 \mathrm{~h}$ (NPs in vitro).

Partially purified sap from the Justo cultivar infected by BYDV-PAV/treatment by AuNPs and then treated again in vitro by AuNPs for $24 \mathrm{~h}$ was coded as:

5. Justo-BYDV-PAV/AuNPs (NPs in vivo)-AuNPs$24 \mathrm{~h}$ (NPs in vitro).

Partially purified sap from the Justo cultivar infected by BYDV-PAV/treatment by AuNPs and then treated again in vitro by AuNPs for $48 \mathrm{~h}$ was coded as:

6. Justo-BYDV-PAV/AuNPs (NPs in vivo)-AuNPs$48 \mathrm{~h}$ (NPs in vitro).

\section{Results and discussion}

\section{Characterization of AuNPs and BYDV-PAV particles by TEM}

TEM characterized and revealed the actual size of the prepared AuNPs ranging from sizes 3.151 and $31.67 \mathrm{~nm}$ with a final concentration of $0.034 \mathrm{mg}$ in $100 \mathrm{ml} \mathrm{d}$. water to have monodispersed spherical AuNPs ranging from 3.151 and $31.67 \mathrm{~nm}$ (Figs. 1a, 2a, 3). Negatively stained BYDV-PAV spherical particles showed measurement about $30 \mathrm{~nm}$ in size in EM (Fig. 1c). In our study, we interfered the bio Nanoparticles (virus) with metal Nanoparticles $(\mathrm{Au})$ in the plant system to find out ability to capture the chemical electron transfer dynamics of the two molecules, which could provide an entirely new perspective on biology (Hong et al. 2015). We selected the most severe BYDV-PAV isolates having spherical morphological capsid that permit high amplification of optical scattering energy in the presence of AuNPs for nano application.

\section{Particle size distribution and zeta potentials}

Zeta sizer chart revealed the concentration with two different dispersed peaks (Fig. 2a) ranging from 3.151 to $31.67 \mathrm{~nm}$ in the width of two peaks (1-100 nm) having different intensities of $75.3 \%$ for the former and $24.7 \%$ for the latter. The zeta potential chart (Fig. 2b) had three negative heteros different, 10.8, 31.0 and $54.0 \mathrm{mV}$, respectively, in the solutions. According to spectrophotometry of BYDV-PAV from purified virus preparations, the average of virus yield was $0.62 \mathrm{mg} / \mathrm{ml}$ from $27.30 \mathrm{~g}$ of infected leaves at A260/A280 ratio (Fig. 2c) according to Alkubaisi et al. (2015). Electron micrographs (Fig. 3) illustrated purified AuNPs only that showed well-dispersed particles of size 3.151 and $31.67 \mathrm{~nm}$. The correlation between the zeta potential value and the zeta sizer is inverse proportion. AuNPs solutions are more active and dispersed. The minuscule size of nanoparticles means they exhibit enhanced or different properties when compared with the bulk material. The large surface area of the nanoparticles produces a large volume. Due to the application on leaf surfaces, they enter through the stomata openings or the base of trichomes and translocate to various tissues (Uzu et al. 2010). According to strong electric fields at the surface, the absorption and scattering of electromagnetic radiation by noble metal nanoparticles are strongly boosted. These unique properties supply the strength of styling photothermal cancer therapy. It is desirable to use agents that are dynamic in the beside-infrared (NIR) zone of the radiation spectrum to reduce the light extinction by intrinsic chromophores in native tissue (Huang et al. 2006).

\section{Gold nanoparticles potentially ruin gold barley yellow dwarf virus in cellular plant tissues}

Gold BYDV-PAV particles can exploit the molecular sensing capability of AuNPs optical antennas and the biochemical properties of viral capsids of millions of different capsid topologies. That can terminate the biological hazards and take advantage of using either inherent or endowed biochemical characteristic of the viral capsids to aim subcellular regions (Plummer and Manchester 2013) and in the vivo region. Gold BYDV-PAV particles can potentially function as optical nanosatellites in a biological galaxy, such as cellular tissues and intracellular spaces which exhibit various biomolecular imaging capabilities with detection sensitivity. We propose that the unique and detailed structures of viral capsids can be used as strong plasmonic templates by conjugating novel metals onto their surfaces. The plasmonic metal layer formed in this manner will function as an optical antenna to amplify and convert incident light into confined nanoscale volumes (Hong et al. 2015). Gold viruses can utilize the molecular sensing capability of optical antennas and the biochemical properties of viral capsids ( $\mathrm{Wu}$ et al. 

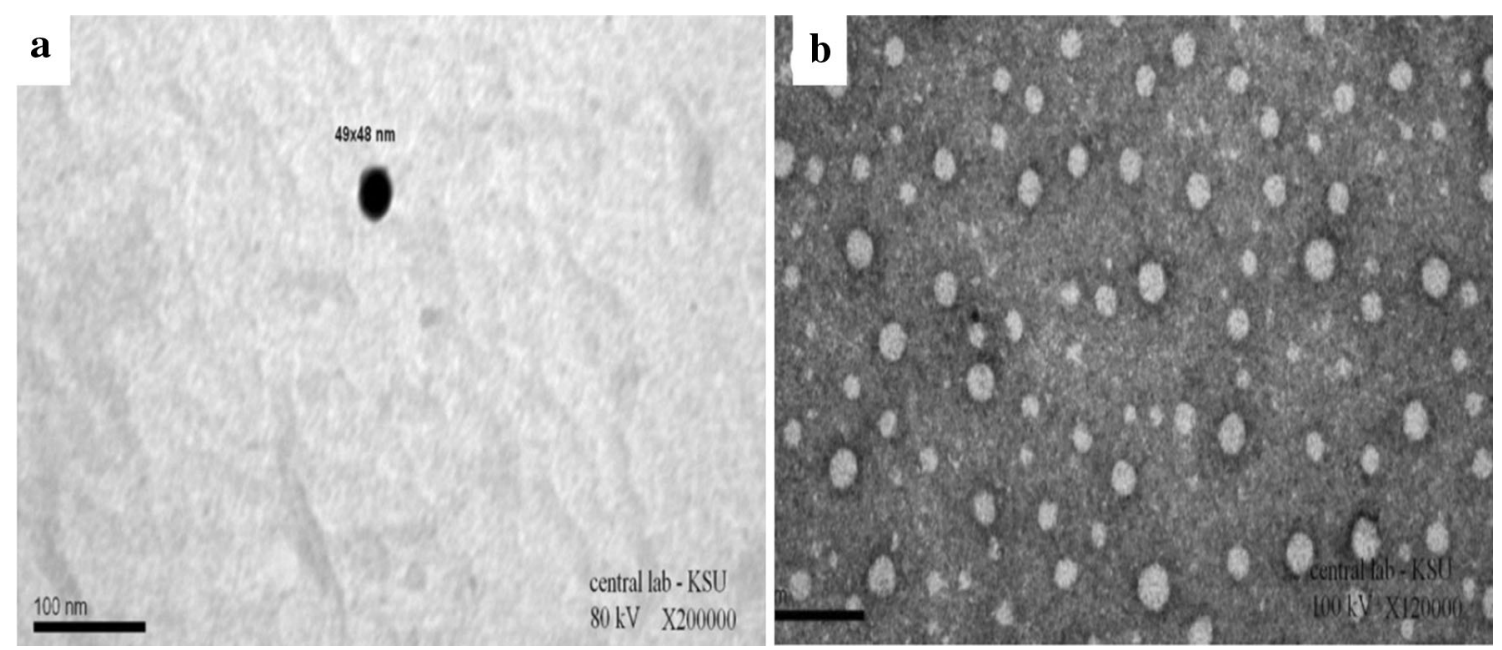

Fig. 1 Electron micrographs illustrated the two studied nanoparticles: a AuNPs, 200000×; b Negatively stained BYDV-PAV particles, $120000 \times$
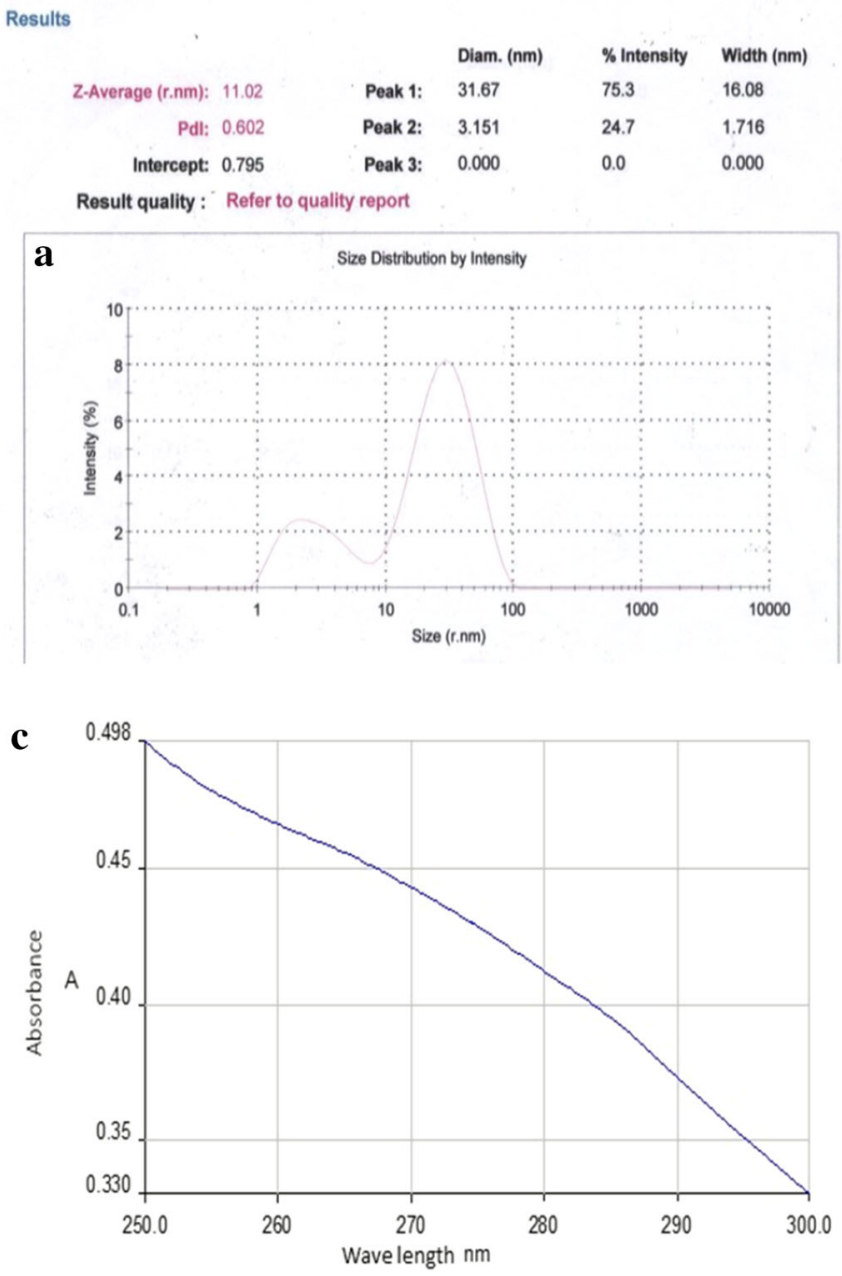

Fig. 2 Characterization of AuNPs and BYDV-PAV particles: a AuNPs have two different peaks appearing in the zeta sizer chart at a range of (1-100) dispersed by their sizes 3.151 and $31.67 \mathrm{~nm}$ with different intensities: $75.3 \%$ for the former and $24.7 \%$ for the latter, b Zeta potential distribution has three hetero different

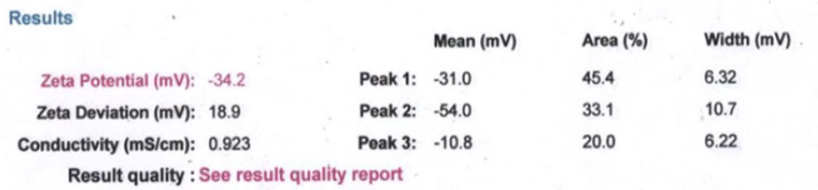

\section{b}

Zeta Potential Distribution

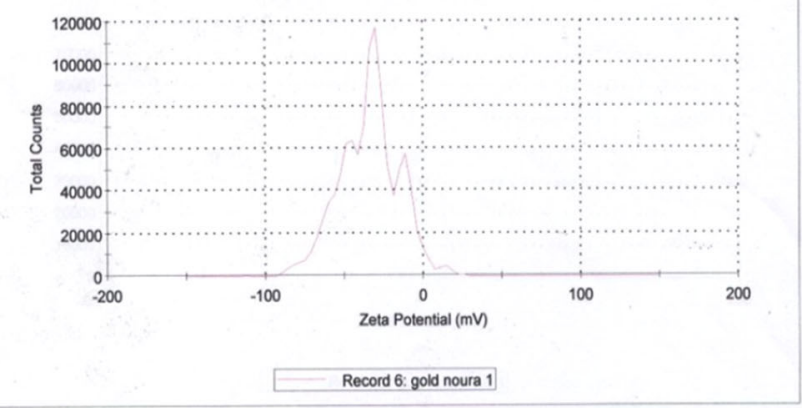

peaks in one limited range from -100 to 0 with negative charge - (10.8, 31.0 and 54.0) $\mathrm{mV}$, respectively; c Ultraviolet absorption spectrum of $27.30 \mathrm{~g}$ leaves from the infected cultivar with ratio A260/ A280 is $0.62 \mathrm{mg} / \mathrm{ml}$ virus yield 


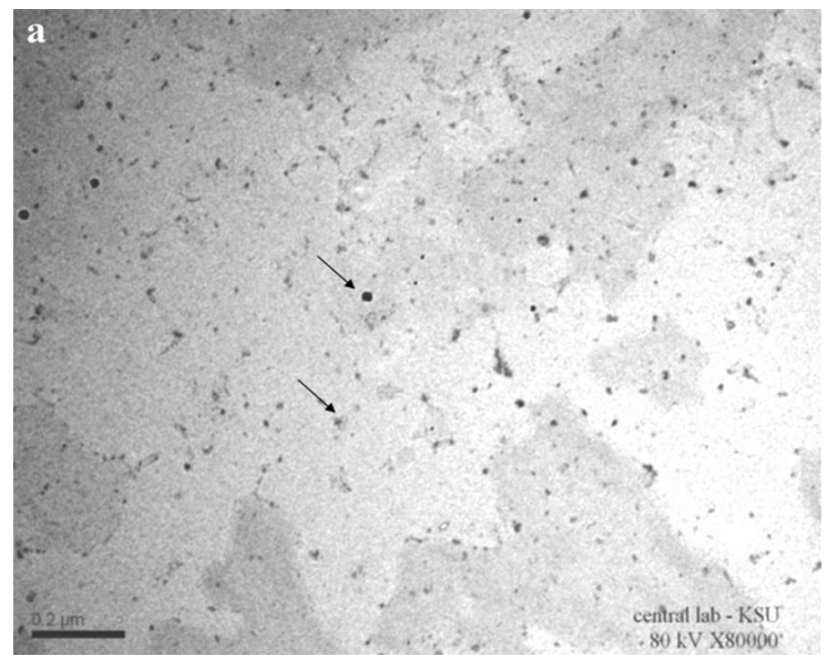

Fig. 3 Electron micrographs illustrated the purified crude sap from barley leaves treated with AuNPs: a AuNPs were noticed to be well dispersed with different sizes ranging from 3.151 and $31.67 \mathrm{~nm}$ (arrows)

2010). The present results illustrate two scenarios of double effects of AuNPs on virus particles inside the plant cells followed by another stress on gold BYDV-PAV particles in vitro in the following comparative studies.

Local-AuNPs/BYDV-PAV (in vivo)-AuNPs-24 vs $48 \mathrm{~h}$ (in vitro) as protective treatment

After $24 \mathrm{~h}$ incubation with AuNPs, numerous deteriorated and ruined VLPs sink in dark AuNPs which had a large

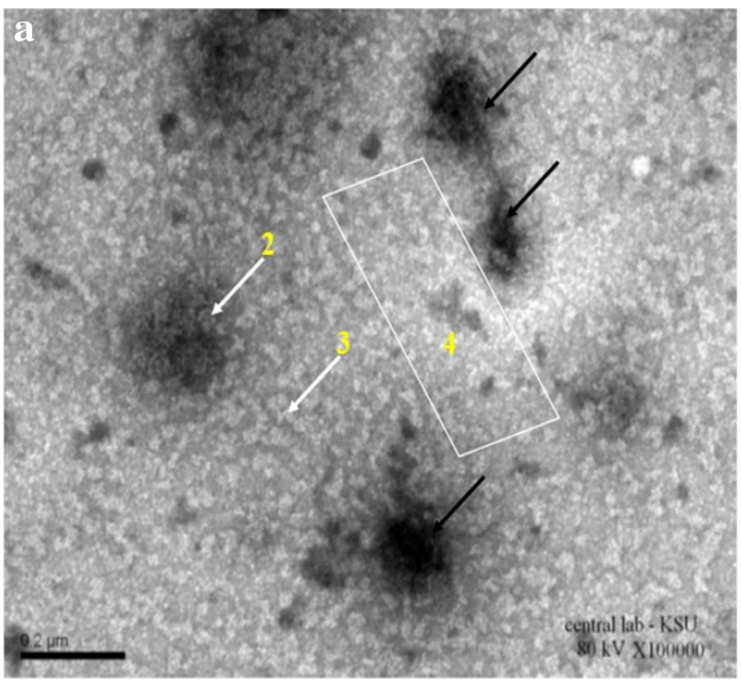

Fig. 4 Electron micrographs illustrated the dual effect of AuNPs for two times processing on virus particles in vivo/vitro according to local-AuNPs/BYDV-PAV (NPs in vivo)-AuNPs-24 vs $48 \mathrm{~h}$ (NPs in vitro): a AuNPs incubation for $24 \mathrm{~h}$ indicated numerous aggregated VLPs, more deteriorated VLPs No. 2 and ruined VLPs No. 3. VLPs sink in a dark black area of AuNPs (black arrows). The large square area of partially vanished VLPs (Fig. 4a). In the $48 \mathrm{~h}$ incubation treatment, the major background of lysed VLPs, as well as numerous ruined corrosive VLPs distributed in most micrographs with dark spots of AuNPs (Fig. 4b).

Justo-AuNPs/BYDV-PAV (in vivo)-AuNPs-24 vs $48 \mathrm{~h}$ (in vitro) as protective treatment

The protective treatment of AuNPs as a first process affecting the virus in vivo followed by more incubation stress of AuNPs for $24 \mathrm{~h}$ indicated numerous individual/ aggregation of more than one puffed VLPs decorated with dark AuNPs and largely circulated area of partially vanished VLPs (Fig. 5a). While AuNPs incubation for $48 \mathrm{~h}$ indicated large white circulated distant with completely lysed anddeteriorated VLPs (Fig. 5b).

\section{Local-BYDV-PAV/AuNPs (in vivo)—AuNPs-24h (in vitro)}

Electron micrographs (Fig. 6a) illustrated that dark areas of the condensed AuNPs attracted VLPs in a massive amount aggregated firmly in spherically deformed groups, which contain ruined and lysed VLPs due to the AuNPs' effectiveness. The damage in purified virus was noticed individually (Fig. 6b). These damaged particles revealed four forms: (1) puffed particles, (2) deteriorated particles decorated with AuNPs, (3) ruined particles, (4) vanished particles.

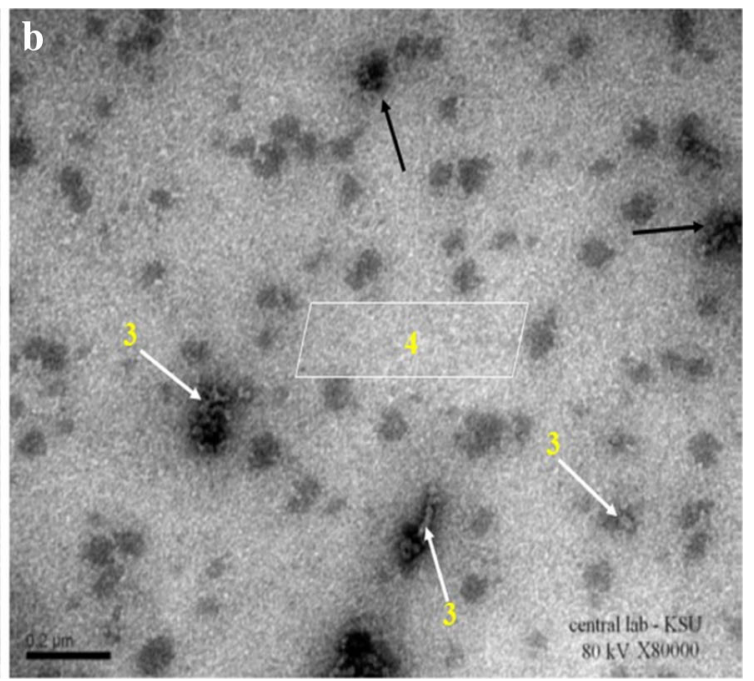

illustrated the area of partially vanished VLPs No. 4; b AuNPs incubation for $48 \mathrm{~h}$ indicated major background square completely lysed VLPs No. 4, as well as numerous ruined corrosive VLPs No. 3 distributed in most micrographs with dark spots of AuNPs (black arrows). Scale bars $0.2 \mu \mathrm{m}$ 


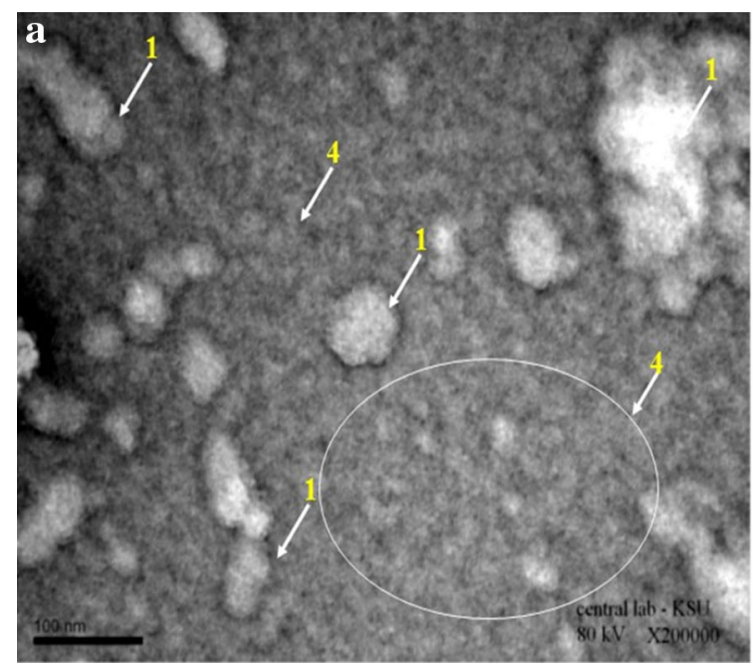

Fig. 5 Electron micrographs illustrated the dual effect of AuNPs for two times processing on virus particles in vivo/vitro according to Justo-AuNPs/BYDV-PAV (NPs in vivo)-AuNPs-24 vs $48 \mathrm{~h}$ (NPs in vitro): a AuNPs incubation for $24 \mathrm{~h}$ indicated numerous puffed and aggregated VLPs No. 1 that decorated with AuNPs. of partially

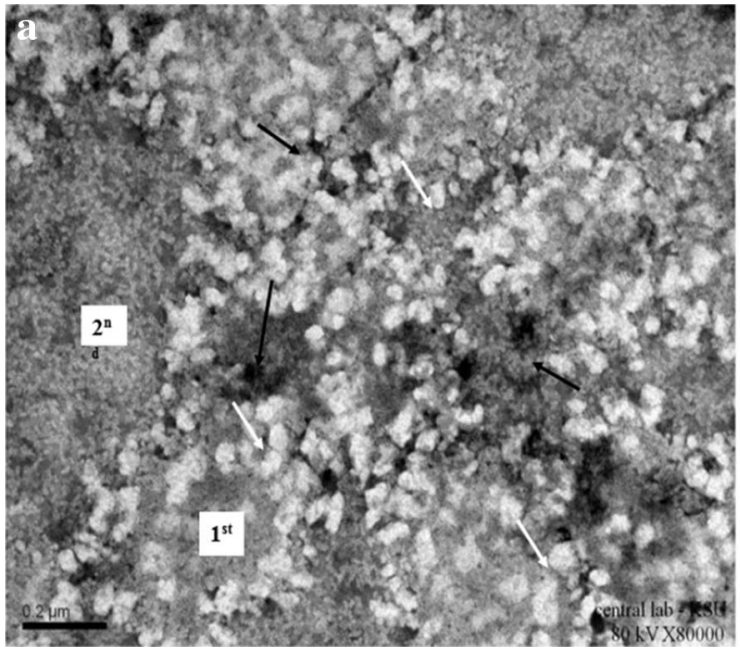

Fig. 6 Electron micrographs illustrated the dual effect of AuNPs for two times processing on virus particles in vivo/vitro according to Local-BYDV-PAV/AuNPs (NPs in vivo)-AuNPs-24 h (NPs in vitro): dark areas of the condensed AuNPs (black arrows) attracted the VLPs (white arrows). VLPs (white arrows) revealed a massive amount of different patches aggregated firmly in spherically deformed groups. These groups were categorized according to the level of damage of viral protein based on the AuNPs effectiveness.

\section{Local-BYDV-PAV/AuNPs (in vivo)-AuNPs-48 $h$} (in vitro)

After $48 \mathrm{~h}$ incubation with AuNPs, the heavy dark area of integrated AuNPs with VLPs led to melting and vanishing VLPs, without any other individual virus particles (Fig. 7a). Variable performance of VLPs was individually visible in four different types of damaged or ruined VLPs: (1) puffed particle, (2) deteriorated particle and those

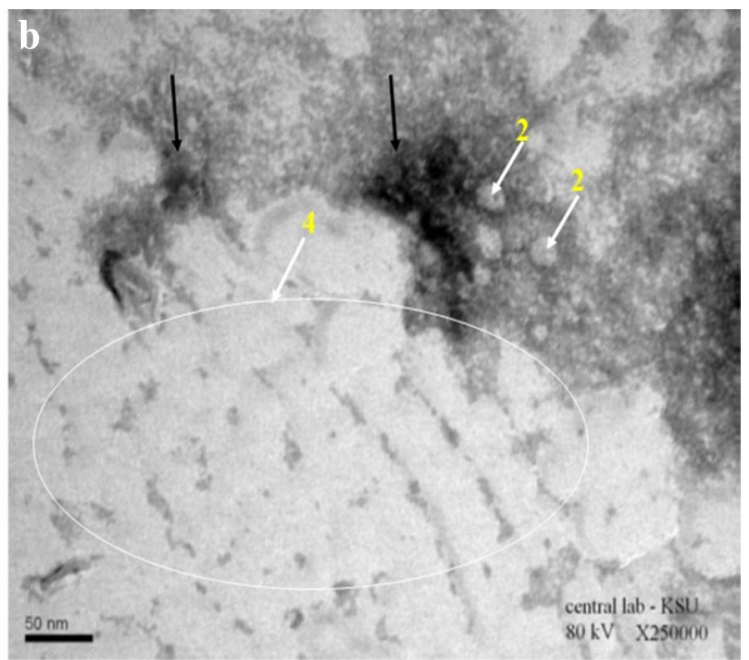

vanished VLPs No. $4.100 \mathrm{~nm}$. Scale bar $100 \mathrm{~nm}$; b AuNPs incubation for $48 \mathrm{~h}$ indicated major white circulated distinguished distant with completely lysed VLPs No. 4, as well as some deteriorated VLPs No. 2 distributed in a lined near dark location of AuNPs (black arrows). Scale bar $50 \mathrm{~nm}$

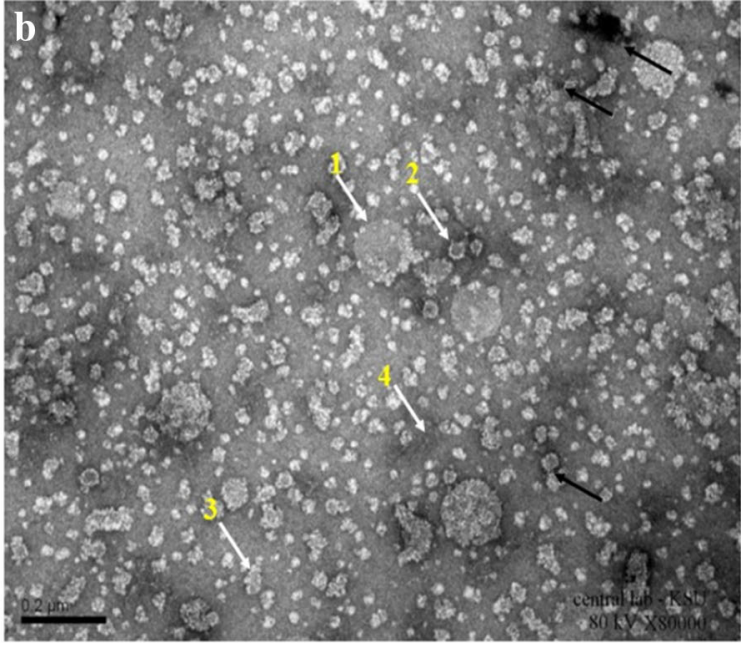

The first level (1st) is ruined VLPs and the second level (2nd) is lysed VLPs. Scale bar $0.2 \mu \mathrm{m}$. b The purified virus was incubated with AuNPs and showed less aggregation of VLPs (white arrows) within AuNPs (black arrows). The yellow numbers indicate the variability of the four major performances of damaged VLPs: 1 puffed particle, 2 deteriorated particle and decorated with AuNPs, 3 ruined particle, 4 vanished particle. Scale bar $0.2 \mu \mathrm{m}$

decorated with AuNPs, (3) ruined particle, (4) vanished particles (Fig. 7b).

Justo-BYDV-PAV/AuNPs (in vivo)-AuNPs-24 h (in vitro)

AuNPs were integrated with some vanished smashed VLPs. Some individual spherical deformed particles disintegrated (Fig. 8a). Figure 8b illustrates clearing and 


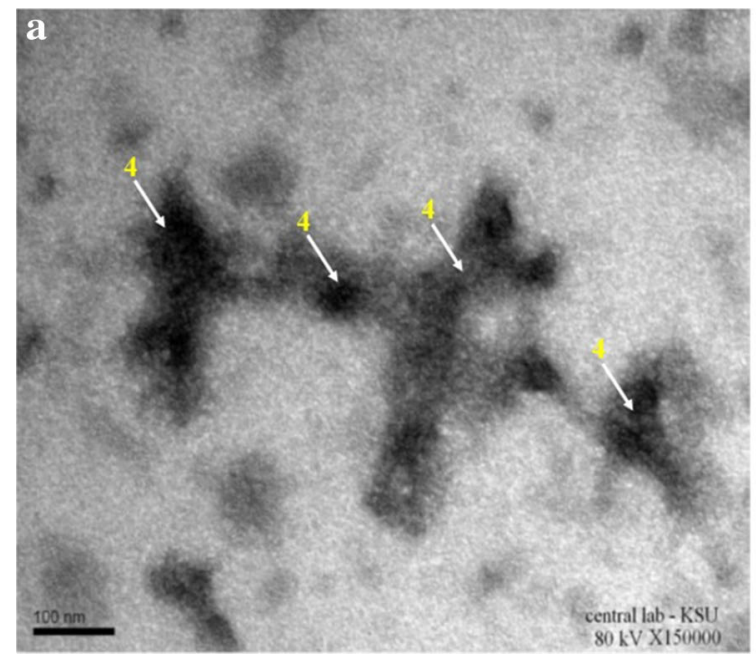

Fig. 7 Electron micrographs illustrated the dual effect of AuNPs for two times processing on virus particles in vivo/vitro according to Local-BYDV-PAV/AuNPs (NPs in vivo)-AuNPs-48 h (NPs in vitro): a AuNPs indicated massive dark area that resembled gathering AuNPs integrated with VLPs giving rise to the (2nd) level of lysed and vanished VLPs (yellow, No. 4). Scale bar $100 \mathrm{~nm}$;

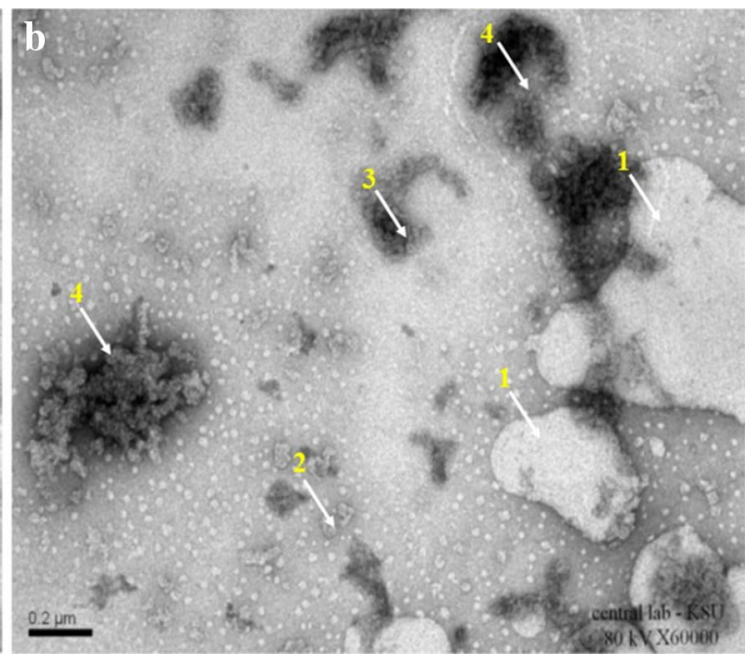

b Purified virus that was incubated with AuNPs. Variable performance of VLPs was individually visible in four different types of damage; ruined VLPs: 1 puffed particle, 2 deteriorated particle and decorated with AuNPs, 3 ruined particle, 4 vanished particles. Scale bar $0.2 \mu \mathrm{m}$

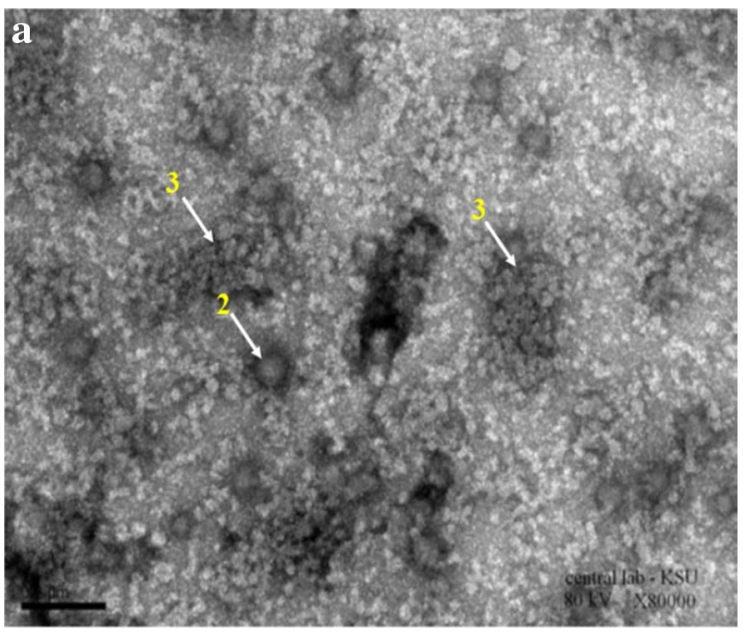

Fig. 8 Electron micrographs illustrated the dual effect of AuNPs for two times processing on virus particles in vivo/vitro according to Justo-BYDV-PAV/AuNPs (NPs in vivo)-AuNPs-24 h (NPs in vitro): a AuNPs indicated the most deteriorated VLPs and ruined No. 2 and 3 appeared in light area (2nd) as a second level, while the dark one of AuNPs integrated with vanished smashed VLPs. Some individual spherical deformed particles disintegrated and decorated No. 2 (yellow) by AuNPs in the black locations. Scale bar $0.2 \mu \mathrm{m}$.

dissolving of VLPs as well as puffed particles. The AuNPs, concentration treatment had the most efficacy on VLPs in both cultivars, while the degree of dispersed AuNPs and its distribution was ideal in Justo. There was a high yield of ruined VLPs in the local cultivar compare to the Justo cultivar for the previous one.

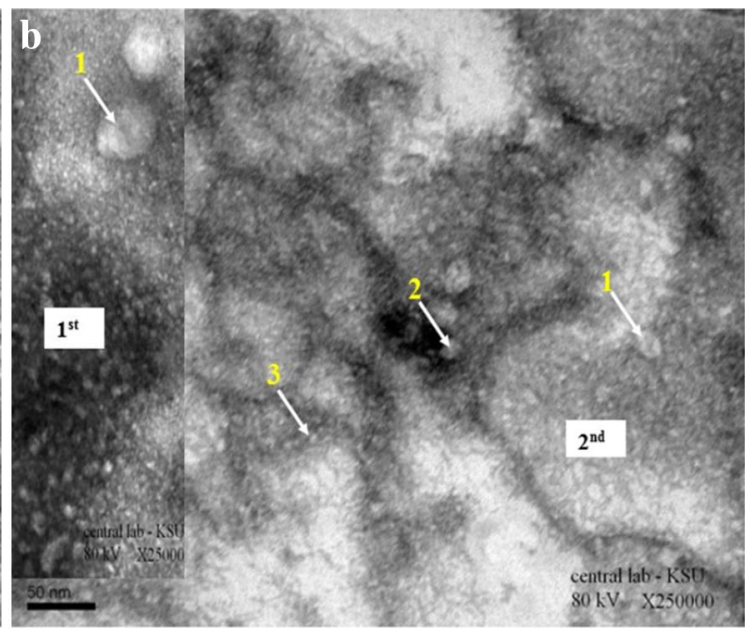

b Purified virus that was incubated with AuNPs treatment showed two different areas that were distinguished and distributed in a wavy dispersed dark look of AuNPs has centered core for grapping three large spherical deteriorated No. 2 of VLPs, while the light area showed clearing and dissolving VLPs. The left upper part of b micrograph revealed puffed particle No. 1 (yellow), while the middle part indicated the first level (1st) of ruined aggregated VLPs in higher magnification. Scale bars (a) and (b) $50 \mathrm{~nm}$

Justo-BYDV-PAV/AuNPs (in vivo)—AuNPs-48 h (in vitro)

After $48 \mathrm{~h}$ incubation with AuNPs, the most puffed VLPs decorated with dark circular AuNPs and deteriorated VLPs which had evacuated parties showed white density 


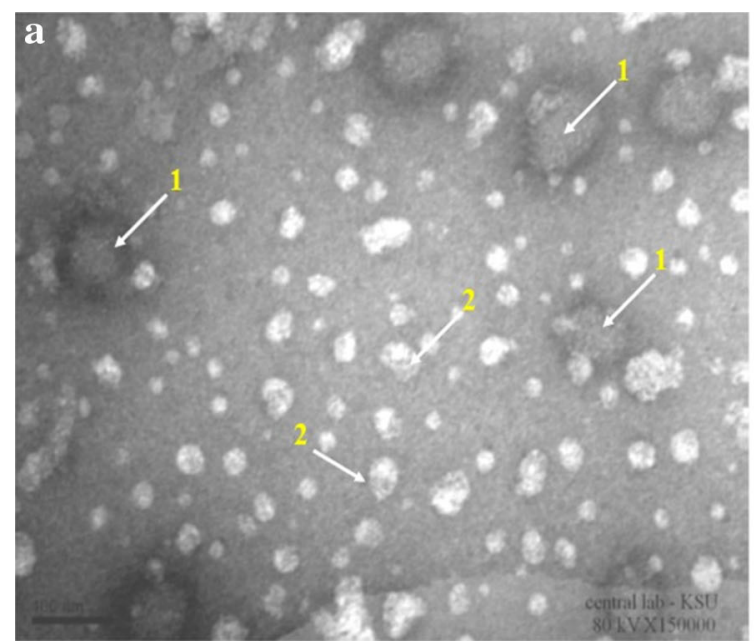

Fig. 9 Electron micrographs illustrated the dual effect of AuNPs for two times processing on virus particles in vivo/vitro according to Justo-BYDV-PAV/AuNPs (NPs in vivo)-AuNPs-48h (NPs in vitro): a AuNPs indicated the most puffed VLPs decorated with dark circular AuNPs and deteriorated VLPs No. 1 and 2. Scale bar $0.2 \mu \mathrm{m}$. b AuNPs incubated with purified virus particles as a dual effect that showed two different areas. These were distinguished and

(Fig. 9a). Four puffed spherical VLPs were surrounded by AuNPs and located in the same area of dissolving VLPs (Fig. 9b). Previous studies using electron microscopy have demonstrated that virus particles have highly ordered natural nano-architectures, with a protein coat called a capsid (Adrian et al. 1984). The outer surface of a viral capsid usually has protrusions and valleys on the subnanometer scale, the accurate the morphology need for optical antennas. Furthermore, viral reproduction yields a remarkably high degree of symmetry and uniformity that is beyond the capability of current nanotechnology. It was greater in the local cultivar. compared to the Justo cultivar. These features make viral nanoparticles attractive templates for optical antennas of AuNPs in our study. Additionally, infection selectivity was remarkable in the local cultivar. Moreover, elimination of harmful viral effects has enabled virus resistance. The amount of nanoparticle accumulation in plants varies with the reduction potential of ions and the reducing capacity of plants that depends on the presence of various polyphenols and other heterocyclic compounds present in plants. No single barley variety is resistant or susceptible to all the great diseases. Thus, varieties should be selected according to their local adaptability, high yield potential, severe losses in the production and quality of wheat and barley crops and resistance to the most shared and serious diseases (Douglas et al. 2002a, b). The capsids of most plant viruses are modest and of firm construction, consisting of numerous copies of one or a few sort of protein subunits coordinated with either icosahedral or helical symmetry. In many conditions, capsids are produced in huge amounts either by

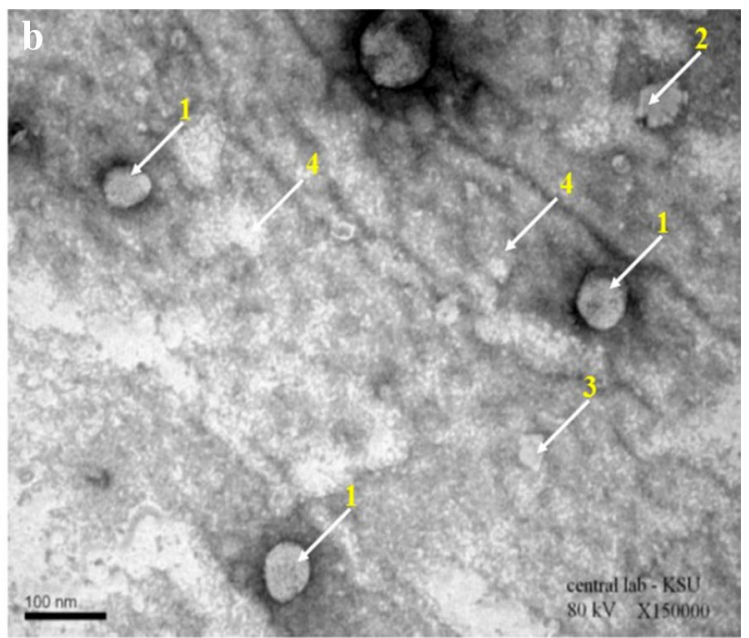

distributed in a wavy dispersed dark look of AuNPs. The centered core attracted three large spherical deteriorated VLPs No. 2. Four puffed spherical VLPs No. 1 surrounded by AuNPs located in the same light area of dissolving VLPs. The effectiveness of AuNPs declared in the four deformed types indicated in the yellow numbers. Scale bar $100 \mathrm{~nm}$

the infection of plants or by the subunit(s) expression of a variety of heterologous systems, given their relative simplicity, stability and ease of production (Lomonossoff and Evans 2011). Thus, the advantage of the morphological characteristics of plant viruses can be taken by integrating AuNPs as a novel metal onto the external surfaces of their capsids for highly sensitive and selective molecular spectroscopic detection such as localized electronic absorption and vibration spectroscopy (Hong et al. 2015). Variable performance of gold capsids was individually visible in four different types of damage or ruined VLPs: (1) puffed particle, (2) deteriorated particle and decorated with AuNPs, (3) ruined particle, and (4) vanished particles (Fig. 7b). Oancea et al. (2009) explain the formation of such chemicals encapsulated in nanocomposites made of layered double hydroxides. In our data, we could relay in this result as a benefit of reverses such phytotoxicity of AuNPs by blending it with virus particles as protein and viral nucleic acid components to accelerate their applicability and effectiveness for virus control. The application of AuNPs particles on the Justo and local cultivars had a great damage on virus particles in TEM (Figs. 4, 5, 6, 7, 8, 9). It could explain the interaction performance in our results which had different degrees between VLPs and AuNPs illustrated as aggregation, surrounding, integration, and accumulation particles. This kind of interval molecular interaction could lead to disappearance or/and swelling, smashing, deformation, corrosiveness and puffiness of infected virus particles. The self-assembly of substratebound monolayers comprised DNA-modified gold nanoparticles. For the first time, bifunctional particles 
containing two independently addressable oligomer sequences were used as building blocks for layer assembly. Saini et al. 2008 proved that Chilo iridescent virus provided a biotemplate which contains a bioscaffold for 2-5 nm of AuNPs on the viral capsid. These a bio scaffold allowed AuNPs to act as nucleation sites for $\mathrm{Au}$ ions in the surrounding solution by allowing electrolysis deposition to occur at the sites. The size of AuNPs has a potential dramatic effect in our study from 3.151 to $66.74 \mathrm{~nm}$. Giljohann et al. 2010 studied the application of AuNPs and found that the size of AuNPs size $(5 \mathrm{~nm})$ could be used as a template for biomimetic high-density lipoprotein structure which makes this size control necessary. Cheung et al. (2003) Proved in this respect that most viruses are in the scale of nanometers, and supermolecules and assembly viruses are of tens of nanometers in size. The AuNPs size ranged between 10 and $40 \mathrm{~nm}$ as shown in Fig. 1. Moreover, nanometric building blocks can aid in the survey of the molecularly directed assembly of biomolecular arrays. Hilger et al. 2002 add an alternating magnetic field that could also be used to heat the magnetic nanoparticles, thus making hyperthermia treatment of tumors feasible and a potential simultaneous treatment. This strategy of AuNPs application in our study induced by combining virus particles with AuNPs possibly leads to synergistic therapeutic effects for inducing virus resistance by the interaction performances, as illustrated in TEM. Giljohann et al. (2010) studied the modified AuNPs for inducing gene regulation upon entry into the cytoplasm of the cell; the DNA-AuNPs can bind to the strand preventing translation of the mRNAs corresponding protein. Different sizes of particles are easily distinguishable in electron micrographs, allowing simultaneous multiple labeling experiments In vitro analysis, AuNPs, are used to locate and probe viruses. In vivo applications of gold nanoparticles illustrated procedures for probing as well as diagnosis and hyperthermic treatment that allow the targeted destruction of cells. There is an important key point in the model he explained related to $\mathrm{Tm}$ of nucleic acid. The oligonucleotides on the AuNP surface are close enough such that the counterions associated with one oligonucleotide also act to screen the negative charge on adjacent oligonucleotides. This additional cost screening results in greater oligonucleotide duplex stabilization about free DNA strands and explains why the DNA-AuNP aggregates melt at higher temperatures than the same unconjugated oligonucleotide duplexes under the same conditions. Patel et al. (2007) cleared that nuclear localization signal NLS peptide is a virus-derived protein fragment that interacts with intracellular proteins for transport across the nuclear envelope. Goldfarb et al. (1985) interpreted that addition of NLS peptide to the DNA-AuNP did not change the binding properties of the oligonucleotides, but it did alter the intercellular localization of the AuNPs. The way of AuNPs application on the plant was performed in vivo, in-vitro and with both of them The most efficient ones on virus particles are those treated by dual stress effect of AuNPs (Figs. 4b, 5 b, 7, 9). Nair et al. (2010) illustrated the mechanism of AuNPs inside the plant. They reported that the formation of nanoparticles, whether they are formed outside in the media and then translocated to plants or whether they conformed with the reduction of metal salts within the plants itself, still needs more clarification. The in vitro assembly of empty virions has been extensively characterized, and these protein cages have great potential to be utilized as constrained reaction vessels for material synthesis and/or entrapment in the field of nanotechnology (Douglas et al. 2002a, b). Recently, plant virus capsids as a protein shell that forms the surface of a typical plant virus particle are useful templates. All virus capsids were assembled from virus-coded protein subunits called capsomeres. Many plant viruses assemble capsids with precise 3D structures providing nanoscale architectures that are highly homogeneous and can produce a great mass. Capsids are adjustable to both genetic and chemical modifications, allowing new functions to be integrated into their structure by the layout. The three capsid appearances, at the interior surface, the outer surface, or the interface between coat protein subunits, can be separately functionalized to produce multifunctional templates (Young et al. 2008). Plant virus-like particles (VLPs) have attracted attention as potent reagents for bionanotechnology. Plant virus-like particles (VLPs) have attracted attention as potent reagents for bio nanotechnology science having genetic materials and chemical alteration of super protein molecular structures (Lomonossoff and Evans 2011). In our study, we obtained four biomolecule alterations of VLPs due to dual AuNPs probing illustratedin Fig. 6b. Homogeneity of virus morphologies in nature and high-throughput reproducibility accelerate the potential for the use of viral particles in optical sensing applications. Moreover, using asymmetric gold coupling leaves parts of the native capsid uncovered that can interact with host cells and biochemically alter functions. Such gold virus nanoparticles can metaphorically work as 'nanosatellites' exploring 'cellular galaxies' (Hong et al. 2015). Therefore, biological structures and biomolecules are promising tools for bionanotechnological applications. The study subject of the area here is to probe BYDV-PAV with AuNPs to diminish its infectivity hazards in plant system and coupling these purified asymmetric gold virus particles once more with AuNPs in vitro to follow up morphological alteration of these gold BYDVPAV nanostructures as a result of dual AuNPs stress in vivo and in vitro as nanosatellites. 


\section{Conclusion}

AuNPs and biocompatible nature characters of BYDV nanoparticles have been postulated in the present study. In vivo conditions, sustained effect of AuNPs inside the plant system will have strong anti-virus influence on viral protein. Hence, this nanoparticle may perhaps be recommended as therapeutics while treating plants infected with the virus.

Acknowledgements This research project was supported by a grant from the "Research Center of the Center for Female Scientific and Medical Colleges," Deanship of Scientific Research, KSU.

\section{Compliance with ethical standards}

Conflict of interest The authors declare that they have no competing interest.

Open Access This article is distributed under the terms of the Creative Commons Attribution 4.0 International License (http:// creativecommons.org/licenses/by/4.0/), which permits unrestricted use, distribution, and reproduction in any medium, provided you give appropriate credit to the original author(s) and the source, provide a link to the Creative Commons license, and indicate if changes were made.

\section{References}

Adrian M, Dubochet J, Lepault J, Mcdowall AW (1984) Cryoelectron microscopy of viruses. Nature 308:32-36

Alkubaisi NA, Aref NMA, Hindi AA (2015) Method of inhibiting plant viruses using gold nanoparticles. US Patent 9198434 B1

Capek I (2015) Viral nanoparticles, noble metal decorated viruses and their nanoconjugates. Adv Colloid Interface Sci 222:119-134

Cheung CL, Camarero JA, Woods BW, Lin T, Johnson JE, De Yoreo JJ (2003) Fabrication of assembled virus nanostructures on templates of chemoselective linkers formed by scanning probe nanolithography. J Am Chem Soc 125(23):6848-6849

Douglas T, Allen M, Young M (2002a) Self-assembling protein cage systems and applications in nanotechnology. In: Steinbüchel A (ed) Biopolymers. Wiley, Weinheim, pp 405-426

Douglas T, Strable E, Willits D, Aitouchen A, Libera M, Young M (2002b) Protein engineering of a viral cage for constrained nanomaterials synthesis. Adv Mater 14:415-418

FAO (Food and Agriculture Organization of the United Nations) (2011) Agriculture, food, and water nanotechnologies for the poor, opportunities and constraints

Giljohann DA, Seferos DS, Daniel WL, Massich MD, Patel PC, Mirkin CA (2010) Gold nanoparticles for biology and medicine. Angew Chem Int Ed 49(19):3280-3294

Goldfarb DS, Gariépy J, Schoolnik G, Kornberg RD (1985) Synthetic peptides as nuclear localization signals. Nature 322(6080):641-644
Hassellöv M, Readman JW, Ranville JF, Tiede K (2008) Nanoparticle analysis and characterization methodologies in environmental risk assessment of engineered nanoparticles. Ecotoxicology 17(5):344-361

Hilger I, Hiergeist ROBERT, Hergt R, Winnefeld KLAUS, Schubert HARALD, Kaiser WA (2002) Thermal ablation of tumors using magnetic nanoparticles: an in vivo feasibility study. Invest Radiol 37(10):580-586

Hong S, Lee M-Y, Jackson AO, Lee LP (2015) Bioinspired optical antennas: gold plant viruses. Light Sci Appl 4(3):e267

Huang X, El-Sayed IH, Qian W, El-Sayed MA (2006) Cancer cell imaging and photothermal therapy in the near-infrared region by using gold nanorods. J Am Chem Soc 128(6):2115-2120

Jarošová J, Chrpová J, Šíp V, Kundu JK (2012) A comparative study of the Barley yellow dwarf virus species PAV and PAS: distribution, accumulation, and host resistance. Plant Pathol 62(2):436-443

Li K, Nguyen HG, Lu X, Wang Q (2010) Viruses and they are potential in bioimaging and biosensing applications. Analyst 135(1):21-27

Lomonossoff GP, Evans DJ (2011) Applications of plant viruses in bionanotechnology. Plant viral vectors. Springer, Berlin, pp 61-87

Nair R, Varghese SH, Nair BG, Maekawa T, Yoshida Y, Kumar DS (2010) Nanoparticulate material delivery to plants. Plant Sci 179(3):154-163

Niemeyer CM (2001) Nanoparticles, proteins, and nucleic acids: biotechnology meets materials science. Angew Chem Int Ed 40(22):4128-4158

Niemeyer CM, Ceyhan B, Noyong M, Simon U (2003) Bifunctional DNA-gold nanoparticle conjugates as building blocks for the self-assembly of cross-linked particle layers. Biochem Biophys Res Commun 311(4):995-999

Oancea E, Vriens J, Brauchi S, Jun J, Splawski I, Clapham DE (2009) TRPM1 forms ion channels associated with melanin content in melanocytes. Sci Signal 2(70):ra21

Patel LN, Zaro JL, Shen W-C (2007) Cell-penetrating peptides: intracellular pathways and pharmaceutical perspectives. Pharm Res 24(11):1977-1992

Plummer EM, Manchester M (2013) Endocytic uptake pathways utilized by CPMV nanoparticles. Mol Pharm 10:26-32

Saini V (2008) Adenovirus as a platform for assembly and targeted delivery of gold nanoparticles to tumor cells (Doctoral dissertation, The University of Alabama at Birmingham)

Steinmetz NF, Evans DJ (2007) Utilization of plant viruses in bionanotechnology. Org Biomol Chem 5(18):2891-2902

Turkevich J, Stevenson PC, Hillier J (1951) A study of the nucleation and growth processes in the synthesis of colloidal gold. Discuss Faraday Soc 11:55-75

Uzu G, Sobanska S, Sarret G, Munoz M, Dumat C (2010) Foliar lead uptake by lettuce exposed to atmospheric fallouts. Environ Sci Technol 44:1036-1042

Wu LY, Ross BM, Hong S, Lee LP (2010) Bioinspired nano coral with decoupled cellular targeting and sensing functionality. Small 6:503-507

Young M, Debbie W, Uchida M, Douglas T (2008) Plant viruses as templates for materials and their use in nanotechnology. Annu Rev Phytopathol 46:361-384 